\title{
A rare case of sigmoid volvulus complicating pregnancy in a Tertiary care centre: a case report
}

\section{Rajarajeswari Ramalingam*, Soundarajan Pradiba, Antony Michael}

Department of Obstetrics \& Gynaecology, Thanjavur Medical College, Tamilnadu, India

Received: 19 February 2016

Accepted: 12 March 2016

\section{*Correspondence:}

Dr. Rajarajeswari Ramalingam,

E-mail: drrrrpanju@gmail.com

Copyright: (c) the author(s), publisher and licensee Medip Academy. This is an open-access article distributed under the terms of the Creative Commons Attribution Non-Commercial License, which permits unrestricted non-commercial use, distribution, and reproduction in any medium, provided the original work is properly cited.

\begin{abstract}
Intestinal obstruction complicating pregnancy is an extremely rare complication during pregnancy. Volvulus of the sigmoid colon is the most common cause of intestinal obstruction complicating pregnancy accounting for upto $44 \%$ of the aetiology. We report a case of sigmoid volvulus complicating pregnancy in a woman with 34 weeks amenorrhoea which was diagnosed early in spite of its late presentation and successfully managed with resection and primary anastamosis. Sigmoid volvulus complicating pregnancy carries a high maternal mortality and morbidity, fetal mortality and needs a high index of suspicion and early surgical intervention.
\end{abstract}

Keywords: Sigmoid colon, Volvulus, Pregnancy

\section{INTRODUCTION}

Sigmoid volvulus complicating pregnancy is a rare complication. Since 1885 only 100 similar cases have been reported. ${ }^{1-3}$ The physiologic symptoms of pregnancy may cloud the clinical picture. ${ }^{1,4}$ Sigmoid volvulus complicating pregnancy is an acute surgical emergency which if not intervened immediately carries a high maternal and fetal mortality and morbidity., ${ }^{1,4}$ Laboratory findings are not diagnostic and there is hesitancy in ordering radiological investigations during pregnancy. ${ }^{1,6,7}$ Ultrasonogram may be of help to some extent in the management. ${ }^{8}$ The treatment requires a multidisciplinary approach and surgical intervention is frequently needed. ${ }^{1,8,9}$ The prognosis of sigmoid volvulus during pregnancy is poor. ${ }^{1,4,5}$ Here in we report a case of Sigmoid volvulus complicating pregnancy in a woman with 34 wks of amenorrhoea which was diagnosed early in spite of its late presentation and successfully managed with resection and primary colorectal anastamosis.

\section{CASE REPORT}

A 28 year old primigravida with 34 weeks gestation of an otherwise uneventful pregnancy presented in labour ward causality with complaints of abdominal pain with progressive worsening for the past three days, increasing constipation for three days followed by obstipation for one day and abdominal distension for two days. On physical examination, the patient was febrile, dehydrated, tachypnoiec, tachycardia and hypotension were present with respiratory distress. The abdomen was hugely distended with tenderness, guarding and rigidity with absent bowel sounds. The obstetric examination revealed the uterus to be irritable with fetal heart sounds present. Per vaginal examination revealed a bishop's score of 3/15. Ultrasonogram showed an intra-uterine viable pregnancy with placenta in upper segment. Laboratory investigations was $\mathrm{Hb} 10 \mathrm{~g} \%$, urea $39 \mathrm{mg} \%$, serum creatinine $1.2 \mathrm{mg} \%$, liver function tests normal, serum electrolytes showed acidosis and there was leucocytosis. Plain X-Ray Abdomen revealed dilated gas filled bowel loops. Chest radiographs showed bilateral pneumonitis. Surgeon opinion obtained. Per rectal examination 
revealed rectum to be empty. After initial aggressive resuscitation patient taken up for emergency laparotomy under general anaesthesia with grade IV- E risk.

Peroperatively sigmoid volvulus was seen behind the pregnant uterus (Figure 1). As sigmoid volvulus was found to have undergone ischemic necrosis and nonviable (Figure 2), resection of sigmoid colon was done and primary end to end colorectal anastamosis was performed. Peroperatively 2 units of packed cell transfusion given. Postoperatively patient monitored in intensive care unit and under mechanical ventilation for one day. Broad spectrum antibiotics, antenatal steroids and micronized progesterone were given 48 hours after surgery patient went into labour and delivered an alive preterm female baby $1.7 \mathrm{~kg}$ which was admitted in NICU. The patient had an uneventful recovery and discharged safe on tenth postoperative day.



Figure 1: Pregnant uterus with sigmoid volvulus.

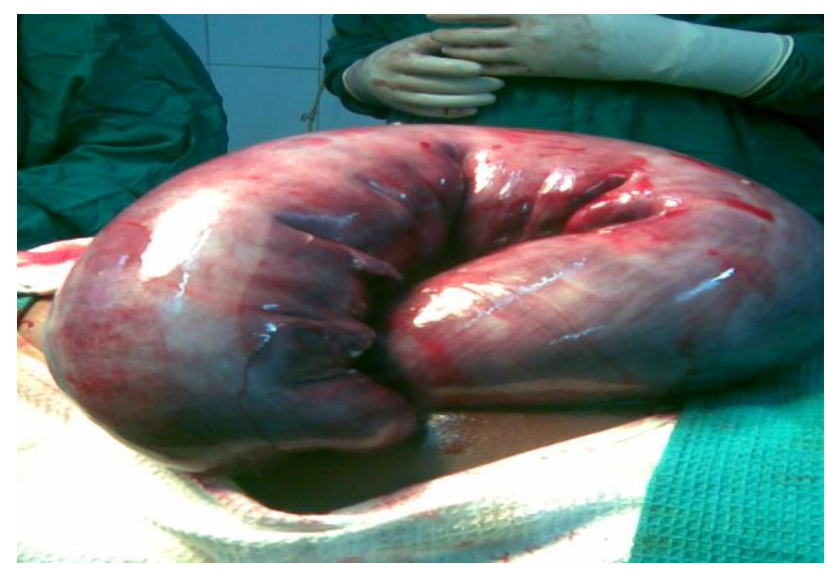

Figure 2: Volvulus necrotic, non-viable.

\section{DISCUSSION}

The incidence of intestinal obstruction during pregnancy is around $1: 1500$ to $1: 66431$ cases. ${ }^{1,5}$ The sigmoid volvulus is the most common cause of intestinal obstruction during pregnancy accounting for $25 \%$ to $44 \%$ of cases. ${ }^{1,5,6,10}$ The mechanism of sigmoid volvulus in pregnancy has been suggested to be due to the displacement of an abnormally mobile sigmoid colon by the enlarging uterus that rise out of the pelvis and can twist around its fixation of point on the sigmoid mesocolon or the pelvic side wall. ${ }^{11,12}$ There is an increased incidence of sigmoid volvulus in the third trimester around $66 \% .^{1,5,6,9,10}$ The typical symptoms of Sigmoid Volvulus in pregnancy known as SV Triad are intermittent and severe abdominal pain, distension and obstipation and the common signs are abdominal tenderness, distension, akinetic bowls sounds and empty rectum. ${ }^{1,4,6,9}$ These typical signs and symptoms though were present in our case, but was thought to be due to pregnancy and hence ignored by the patient. The laboratory findings are not pathognomonic in Sigmoid Volvulus during pregnancy. ${ }^{1,6,7}$ Plain X-ray abdomen showing a horse shoe pattern or coffee bean pattern, carries a risk of radiation to the foetus 0.1 to 0.3 rads. ${ }^{1,13}$ Nevertheless, a nonspecific clinical diagnosis of intestinal obstruction is generally made as was in our case. A definite diagnosis is possible only by MRI or flexible endoscopy. ${ }^{14-17}$ Abdominal and obstetric ultra sonography provides information about the foetus and eliminates or pathologies like Abruptio Placenta, Appendicitis, Cholelithiasis, Acute Pyelonephritis to some extent. ${ }^{1,8}$

The management of Sigmoid Volvulus in pregnancy requires a collaboration of general surgeons, obstetricians and neonatologists. ${ }^{1,8,9}$ The patient should be managed in an intensive care unit with aggressive fluid resuscitation, broad spectrum antibiotics with ventilatory support if the patient is in multiple organ dysfunction as in our patient. Recognition of this entity as an acute surgical emergency and de-compression of proximal bowel is the sine qua non in preventing maternal mortality. ${ }^{5,18}$ The surgical methods can be colonoscopic detorsion, resection of the nonviable colon and colostomy or end to end anastamosis. Flexible colonoscopic detorsion is difficult in late pregnancy due to mechanical impediment by gravid uterus, but successful detortion can be performed in non-gangrenous sigmoid volvulus of pregnancy. ${ }^{1,9,17}$

When an emergency surgical intervention is required in patients with sigmoid volvulus complicating pregnancy in third trimester, a standard midline incision allows maximal exposure with minimal uterine manipulation. ${ }^{19}$ Usually ischaemic or necrotic bowel is resected, exteriorizing the proximal colon as a terminal colostomy and closing the distal rectum (Hartmann's procedure). ${ }^{20}$ Resection and primary colorectal anastamosis as in our patient is a safe approach with distinct advantage of reduced hospital stay and avoidance of further surgery. ${ }^{1,9,19,21}$ The use of tocolytics and steroids for fetal maturity has to be individualized. When the fetus is alive the surgeon should try to preserve the integrity of the uterus and allow vaginal delivery as in our case. ${ }^{22}$ Emergency LSCS in the presence of multiple organ dysfunction and septicemic shock can develop severe postpartum haemorrhage with $67.7 \%$ mortality which may lead on to hysterectomy and puerperal sepsis. ${ }^{23}$ After surgery the patient may need intensive care in ICU with 
ventilator support because of ARDS as in our patient. ${ }^{23}$ Early suspicion together with prompt intervention with primary anastamosis will minimize maternal/fetal mortality and morbidity as in our patient. ${ }^{1,9,19,21}$

\section{CONCLUSION}

Diagnosis of sigmoid volvulus in pregnancy is a challenge, but a delay in diagnosis increases the rates of feto-maternal mortality. Studies have shown that in sigmoid volvulus complicating pregnancies the prognosis is poor with 12 to $24 \%$ maternal mortality and 20 to $26 \%$ foetal mortality. A high incidence of clinical suspicion and timely surgical intervention are the key to a favourable outcome.

\section{Funding: No funding sources}

Conflict of interest: None declared

Ethical approval: Not required

\section{REFERENCES}

1. Atamanalp SS, Ozturk G. Sigmoid volvulus in pregnancy. Turk J Med Sci. 2012;42:9-15.

2. Weingrow D, McCague A, Shah R, Laiezarzadeh F, Delayed presentation of sigmoid volvulus in a young woman. West J Emerg Med. 2012:13(1):100-2.

3. Raveenthiram V, Matiba TE, Atamanalp SS, De U. Volvolus of the sigmoid colon. Colorectal Dis. 2010;12(7):e1-17.

4. Aishawi JS. Recurrent sigmoid volvulus in pregnancy; report of a case and review of the literature. Dis Colon Rectum. 2005;48:1811-3.

5. Perdue PW, Johnson HW Jr, Stafford PW. Intestinal obstruction during pregnancy. Am J Surg. 1992;164:384-8.

6. De U, De KK, Sigmoid volvulus complicating pregnancy. Indian J Med Sci. 2005;59:317-9.

7. Hofmeyr GJ, Sonnendecker EWW. Sigmoid volvulus in advanced pregnancy. Report of 2 cases. S Afr Jed J. 1985;67:63-4.

8. Vo TM, Gyaneshwar R, Mayer C. Concurrent sigmoid volvulus and hernation through broad ligament defect during pregfnancy: A case report and literature review. J Obstet Gynaecol. 2008;34:65862.

9. Atamanalp SS. Sigmoid volvulus complicating pregnancy. A case report Eurasian J Med 2015;47:75-6.

10. Kolusari A, Kurdoglu M, Adali E. Sigmoid volvulus in pregnancy and puerperium: a case series. Cases Journal. 2009;2(9):9275.
11. Ballantyne GH, Brandner MD. Volvulus of the colon. Incidence and mortality. Annals of Surgery. 1985;202(1):83-92.

12. Alshawi JS. Recurrent sigmoid volvulus in pregnancy: report of a case and review of the literature. Diseases of the Colon and Rectum. 2005;48(9):1811-3.

13. Karam PA. Determining and reporting fetal radiation exposure from diagnostic radiation. Health Phys. 2000;79:S85-90.

14. Atamanalp SS. Sigmoid Volvulus; diagnosis in 938 patients over 45.5 years. Tech Coloproctol. 2013;17:419-24

15. Atamanalp SS, Kantarct M, Ayclinli B. The diagnostic efficiency of CT and MRI in ileosigmoid knotting. Turk J Med Sci. 2014;44:1134-5.

16. Palmucci S, Lanza ML, Gulino F, Scilletta B. Daignosis of a sigmoid volvulus in pregnancy: ultrasonography and magnetic resonance imaging findings. J.Radiol Case Rep. 2014;8(2):54-62.

17. Aftab Z, Toro A, Abdelaal, Dasovky M, Gehani S, Abdul Mola A. Endoscopic reduction of a volvulus of the sigmoid colon in pregnancy: case report and a comprehensive review of the literature. World $\mathbf{J}$ Emerg Surg. 2014;9:41.

18. Safioleas M, Chatziconstantinou C. Clinical considerations and therapeutic strategy for sigmoid volvulus in the elderly: a study of 33 cases. World Journal of Gastroenterology. 2007;13(6):921-4.

19. Machado NO, Machado L. Sigmoid volvulus complicating pregnancy managed by resection and primary anastamosis. Case report with literature review. Sulthan Qaboos University Medical Scienses Journal. 2009;9:84-8.

20. Al Maksoud AM, Barsoum AK, Moneer MM. Sigmoid volvulus during pregnancy; A rare nonobstetric complication. Report of a case and review of the literature. Int J Surg Case Repl. 2015;17:61-4.

21. Redlich A, Rickes S, Costa SD. Small bowel obstruction in pregnancy. Archives of Gynecology and Obstetrics. 2007;275(5):381-3.

22. Fraser JL, Eckert La. Volvulus complicating pregnancy. Canadian Medical Association Journal. 1983;128(9):1045-8.

23. Perez Assef A, Acevedo Rodriguez O. Characterization of obstetric patients with multiple organ failure in the intestive care unit of a Havana Teaching Hospital, 1998 to 2006. MEDICC Review. 2010;12(2):27-32.

Cite this article as: Ramalingam $\mathrm{R}$, Pradiba $\mathrm{S}$, Michael A. A rare case of sigmoid volvulus complicating pregnancy in a Tertiary care centre: a case report. Int J Reprod Contracept Obstet Gynecol 2016;5:1273-5. 